\title{
Competencias digitales y resiliencia: una revisión teórica enfocada en el profesorado
}

\section{Digital competences and resilience: a theoretical review focused on teachers}

\author{
Jhon Holguin-Alvarez, ${ }^{\text {a }}$ Milagritos Rodríguez Rojas, ${ }^{2}$ Rosa M. Romero-Hermoza, ${ }^{3}$ \\ Fernando Ledesma-Pérez, ${ }^{4}$ Juana Cruz-Montero ${ }^{5}$ \\ Universidad César Vallejo, Perú ${ }^{12345}$
}

(iD) ORCID ID: https://orcid.org/0000-0001-5786-0763 ${ }^{1}$

(iD) ORCID ID: https://orcid.org/0000-0002-8873-1785

(iD) ORCID ID: https://orcid.org/0000-0002-5129-3160 3

(iD ORCID ID: https://orcid.org/0000-0003-4572-13814

ID ORCID ID: https://orcid.org/0000-0002-7772-6681

Recibido: 15 de marzo de 2021

Aceptado: 08 de julio de 2021

\begin{abstract}
Resumen
Las competencias digitales y la resiliencia son destrezas o capacidades funcionales para la humanización tecnológica y el soporte emocional para integrarse a la ciudadanía digital. En el campo educacional, exclusivamente, en el profesorado sirven como herramientas sostenibles para la educación virtual. El objetivo de la investigación es revisar los fundamentos de las competencias digitales y resiliencia. La metodología es cualitativa de tipo revisión narrativa de literatura. Revisamos 101 artículos, de los cuales compilamos 49 documentos científicos bajo criterios de selección de la literatura teórica, empírica y sistemática. Los resultados concluyen que las competencias digitales se enfocan en el Conectivismo como Constructivismo Digital, en el cual se comprende que los docentes conocen y utilizan los medios y la información de las redes para realizar procesos formativos. Por otro lado, la teoría humanista y ecologista de la resiliencia la establecen como la competencia del docente para superar las debilidades e incrementar las fortalezas en su tarea virtual.
\end{abstract}

Palabras Claves: Competencias Digitales; Educación Remota; Educación Virtual; Empoderamiento Digital; Resiliencia.

\footnotetext{
Abstract

Digital competences and resilience are functional skills or capacities for technological humanization and emotional support to integrate into digital citizenship. In the educational field, exclusively, in the teaching staff they serve as sustainable tools for virtual education. The objective of the research is to review the fundamentals of digital skills and resilience. The methodology is qualitative, of a narrative literature review type. We reviewed 101 articles, of which we compiled 49 scientific documents under selection criteria from the theoretical,

E-mail: jholguin@ucv.edu.pe; jhonholguinalvarez@ucv.edu.pe
} 
empirical and systematic literature. The results conclude that digital competences focus on Connectivism as Digital Constructivism, in which it is understood that teachers know and use the media and information of the networks to carry out training processes. On the other hand, the humanist and ecological theory of resilience establishes it as the teacher's competence to overcome weaknesses and increase strengths in their virtual task.

Keywords: Digital Competences; Digital Empowerment; Remote Education; Resilience; Virtual education.

\section{Introducción}

La importancia del estudio de revisión, radica en recopilar, analizar y comparar las propuestas teóricas que conforman los constructos Competencias digitales y Resiliencia, así también los resultados sobre estas variables relacionadas a sus respectivas problemáticas sociales y educativas. A dicho contexto se agrega otro más, el de pandemia, en el cual se erigen la educación remota y el uso de entornos de virtualidad, por lo que consideramos que el docente o profesor necesita de estas competencias para utilizar la información (Consejo de la Unión Europea en Sánchez-Cruzado et al., 2021; Tondeur et al., 2015), aplicar cambios sobre ella y utilizarla para los fines educativos necesarios en las comunidades escolares o universitarias. Esto relevante, mucho más, ante la realidad pandémica, de la cual se sabe que el problema coyuntural respecto a redes, se supo que entre 5 y $6 \%$ de profesores han desarrollado competencias digitales bajo modalidad de capacitación virtual (Minedu, Escale, 2019). Así también, el problema respecto al aumento de defunciones entre el año 2020 y 2021 es significativo, ya que la cifra de fallecidos se incrementó de 140,000 casos de contagios en mayo de 2020 a 2, 033,606 en junio de 2021 (Minsa, Sala Situacional Covid-19, Perú). Sin embargo, solo se han desarrollado $5 \%$ de 50 estrategias válidas para desarrollar el componente emocional durante el transcurso de la pandemia en el mundo (Unesco, 2021).

A la luz de este contexto, es necesario que los educadores conozcan y ejerciten estas competencias, debido a que son consideradas el recurso humano para la integración de las sociedades formativas. Ante esta acepción, habría que entender a las escuelas como sociedades formativas, lo cual exige fortalecer las competencias del profesorado (De Jager et al., 2017), generar procesos de gestión educativa que permitan complementar el bienestar de esta población, y así brindar su servicio con calidad, sin embargo, este bienestar significa incrementar sus habilidades digitales para permitir desarrollar procesos pedagógicos y de investigación más sustentables, así como también potenciar su tranquilidad personal mediante la capacidad de resiliencia (Mithani et al., 2020; Wingerden \& Poell, 2019), ante de ello conocer su concepto y los hallazgos empíricos de impacto. 
Los estudios han reportado que las competencias digitales están asociadas al apoyo de la gestión institucional (Instefjord et al., 2017; Lucas et al., 2021; Tondeur et al., 2015), aunque la eficacia [en el proceso de enseñanza] también está relacionada a esta variable, pero con menor impacto (Instefjord et al., 20217). Esto redundaría en lo hallado por Hämäläinen et al. (2021), al mencionar que el apoyo en la gestión de las competencias digitales también se transfiere al alumnado a cargo del profesorado, por cuanto se desarrolla el uso de tecnologías en los estudiantes como un co-aprendizaje y empoderamiento. Algo similar se ha encontrado en un estudio nacional (Holguin-Alvarez et al., 2021b), la transferencia de competencias digitales se logra durante la educación básica, en la formación universitaria o en la interacción social. Se debe aceptar que se necesita mayor inversión en las tecnologías más actuales para impulsar al uso multidisciplinario en el grupo de docentes de toda escuela (Lucas et al., 2021). También se evidencian relaciones inversas entre la resiliencia y la depresión en personas relacionadas al Covid-19 (Villalobos-Otayza et al., 2021).

No obstante, debemos aceptar que el concepto de competencia digital se ha elaborado hasta el momento sobre los cimientos de la Comisión de la Unión Europea bajo el marco DigComp (Hämäläinen et al., 2021; Lucas et al., 2021; Wild \& Schulze, 2021; Zhao et al., 2021). El docente contemporáneo debe presentar mejores habilidades en su perfil profesional, debido a dos factores esenciales emergentes: (a) crecimiento en la aparición de tecnologías, (b) desarrollo de nuevas metodologías basadas en la inteligencia artificial, por lo que su respuesta debe ser ávida en relación a los contextos principales de atención, lo cual permite aceptar que las competencias digitales son el grupo de habilidades, conocimientos y actitudes en el uso de las tecnologías para ponerlas en práctica, y transferirlas en la mediación educativa ejercida por otro sujeto más hábil (Zhao et al., 2021). Desde esta perspectiva constructiva digital, podemos entender la necesidad del bienestar tanto mediático (digitalización social) y el bienestar resiliente (Instefjord et al.). Prueba de esto son los problemas sociales y económicos que generan disparidad entre los estratos sociales del país.

El bienestar resiliente se encuentra ligado a las competencias digitales. Existe mayor capacidad predictiva en los trabajadores que suelen trabajar organizadamente para percibir los obstáculos en el proceso empresarial (Duchek, 20219). Desde una perspectiva organizacional, entendemos inicialmente a la resiliencia como la capacidad de los sujetos por percibir, evaluar los obstáculos y adaptarse a los problemas bajo la superación de los problemas suscitados en el desarrollo humano y profesional (Ainsworth \& Oldfield, 2019; Duchek, 20219; 2017). Aunque la propuesta sobre resiliencia se sigue construyendo en la perspectiva educacional empresarial/organizacional, es importante revisar su concepto y sus fundamentos para aprender 
a sobrellevar problemas tanto de gestión organizacional como los de gestión educativa, mediante la búsqueda del bienestar psicológico del ser humano (Gu \& Day, 2013; Ungar et al., 2020 Ungar \& Theron, 2019).

También debemos considerar que las instituciones se guían actualmente por procesos distributivos del poder (Andersson et al., 2019), y el entrenamiento institucional que ejercen las organizaciones sobre sus empleados o trabajadores [profesores] (Rupprecht et al., 2019). En este sentido, el concepto de resiliencia se basa en la capacidad de los seres humanos que laboran distribuyendo el poder de gestión o control normativo institucional para lograr la descentralización de los procesos de gestión [distribución del liderazgo]. La docencia en la actualidad, necesita atender de diversas formas a la población estudiantil, la cual comienza a utilizar más contenidos en la red o Internet, recursos de quinta generación y mayor energía y consumo de datos para convivir en medios digitales.

La revisión del constructo de resiliencia influye en las áreas cognitiva y espiritual, para generar en el ser humano: afectividad, autodominio y la seguridad, ante otras variables negativas que aparecen en la pandemia como el aislamiento, la retención emocional y la falta de recursos. Las competencias digitales son habilidades viabilizadoras de la creatividad y de la enseñanza segura en el contexto educativo virtual. El objetivo de investigación es revisar el constructo teórico de las competencias digitales y de la resiliencia desde la perspectiva educacional.

\section{Metodología}

El método de revisión es básica cualitativa, de tipo revisión narrativa de literatura. La revisión permitió la selección, organización y análisis de la literatura fue útil para la elaboración del marco teórico relativo a cada constructo de investigación [competencias digitales, resiliencia] (Hernández et al., 2018). Consideramos criterios de selección de los documentos de investigación revisados, los cuales debían presentar virtudes específicas: (a) Preferencia en el tipo de literatura, (b) origen de la base de datos, (c) orientación temática, (d) idioma [inglés, español, portugués]. En cuanto a los criterios de preferencia en el tipo de literatura de los documentos de investigación (Díaz, 2009; Hernández et al., 2018; Rudas et al., 2013), se priorizaron los artículos de revisión de literatura y aquellos sobre resultados de investigación. Cabe señalar que también se incluyeron para esta selección a los artículos de revisión sistemática. En relación a las bases de datos, consideramos aquellos artículos alojados en revistas indexadas en las bases de datos Scopus, Web of Science, Scielo y DOAJ. También se consideraron referencias de la base de datos Latindex Catálogo 2.0. La búsqueda de algunas 
referencias en Latindex se debió a que en algunos casos se encontraron definiciones dimensionales sobre las variables que complementaban su conceptualización.

Específicamente para la búsqueda de antecedentes empíricos [de resultados], se priorizaron las primeras bases de datos. Una vez seleccionados los documentos, se depuraron los artículos con orientaciones generales o específicas sobre el estudio de otras ciencias no basadas en las perspectivas educativas, en psicología educacional, psicología social, comportamiento humano y psicología positiva (figura 1). En el caso de las competencias digitales se consideraron los enfoques conectivista y construccionista de la educación, para hacer un poco más amplia la búsqueda y selección. Los criterios para la elección de temáticas en resiliencia fueron más amplios, pues se incluyeron algunos dedicados a la resiliencia social, resiliencia en el trabajo y resiliencia en medios digitales, ya que el estudio se dedicó al trabajo docente desde la expectativa laboral, pero sin alejarse de la perspectiva humanística (figura 1).

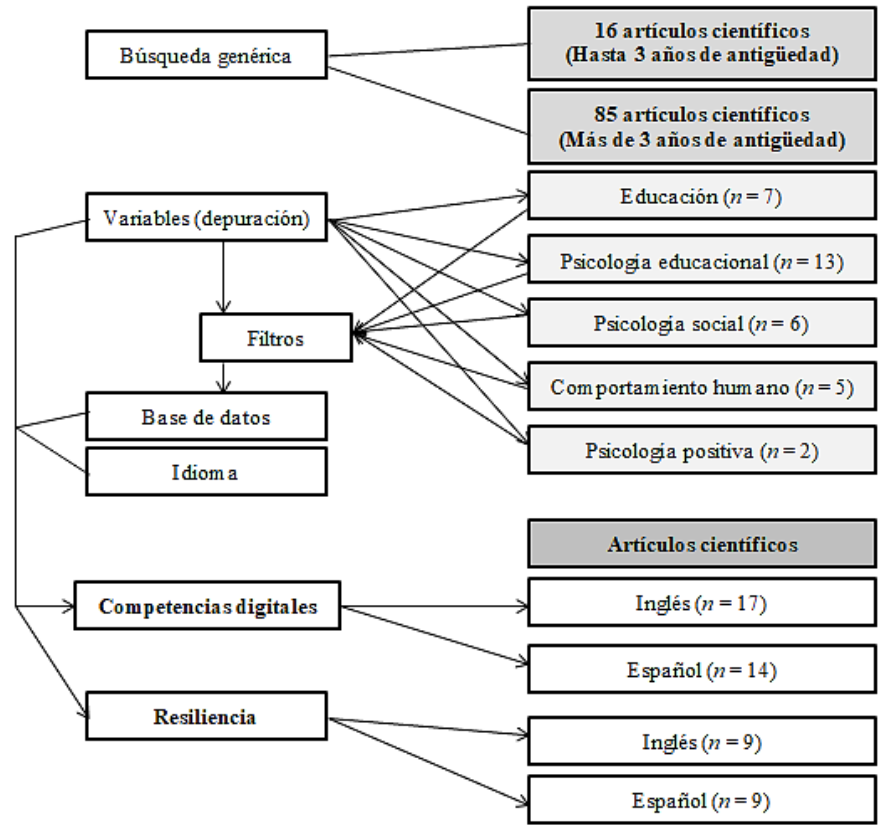

Figura 1. Flujo de selectividad en teorías sobre las competencias digitales y la resiliencia

El tiempo de búsqueda fue de cinco meses de duración. Una búsqueda inicial presentó un total de 2,146 artículos en las bases de datos ScienceDirect y Scopus, con las cuales, encontramos información que podríamos relacionar con las otras bases de datos antes descritas. De acuerdo, a la figura 1, se describe la distribución del inicio de filtración, resultando 101 artículos científicos más relacionados a los enfoques paradigmáticos [conectivismo, constructivismo, cognitivismo...]. Priorizamos el idioma inglés para la búsqueda, pero las 
condiciones de los artículos no fueron equitativas entre sí, siendo los trabajos empíricos sobre ambas variables aquellos publicados en idioma español. Los trabajos en idioma inglés fueron aquellos que incluyeron la descripción de teorías relacionadas, como también sobre la especificación de dimensiones e indicadores sobre los constructos analizados. En razón de los enfoques conectivista y constructivista existió cierta paridad en la literatura hallada, respecto al enfoque humanista se corroboró mayor cantidad de artículos en idioma español, aunque la diferencia fue escasa.

La búsqueda específica se realizó en dos fases (Rudas et al., 2013), aquella en la que se ha encontrado documentos hasta de tres años de antigüedad y otro con mayor a tres. Cabe señalar que para esta búsqueda se utilizaron operadores booleanos de tipo "and" y "or". Por lo cual, se hicieron colecciones de documentos desde términos asociados, en los cuales se utilizaron sinonimias para encontrar términos globales como: "competencia", "habilidad", "destreza", “digital”, "virtual”, "resiliencia”, "resistencia”, "superación”, "humanismo",...entre otros términos. Posteriormente, la depuración de artículos de revisión conceptual y de caracterizaciones dimensionales de las competencias digitales y resiliencia, nos permitió compilar un total de 49 documentos científicos: 31 fueron sobre competencias digitales y 18 sobre resiliencia. En cuanto al idioma, sobre competencias digitales, hallamos 17 artículos en idioma inglés y 14 en español. Sobre resiliencia: 9 en inglés y 9 en español. Respecto a la literatura en relación a los hallazgos empíricos o artículos con resultados científicos, estos fueron 15 sobre competencias digitales y 12 sobre resiliencia.

En cuanto a los enfoques teóricos, se halló un total de 4 artículos con orientación centrada en el conectivismo y en el constructivismo digital. En la clasificación sobre documentos con temática humanista en educación, hallamos un total de 7 artículos de investigación. En una etapa posterior, los artículos seleccionados y depurados fueron clasificados mediante tablas de doble entrada con categorías de autor, año, resultados y conclusiones. Por lo cual, se buscaron similitudes en tanto a los conceptos, las características dimensionales y los hallazgos, y sus diferencias en cuanto a la revisión específica. Para la redacción de datos y la discusión especializada, recurrimos a la generación de mapas mentales, con el fin de compilar los argumentos y resultados más propicios en la redacción del informe de revisión teórica.

\section{Desarrollo de la revisión}

\section{La teoría del conocimiento desde el conectivismo.}


La conectividad entre individuos se vuelve cada vez más intrínseca en las relaciones en redes sociales, como también en los entornos académicos, por lo cual se hace necesaria la consideración de la teoría del conectivismo postulada por Siemens (2004), el sistema de aprendizaje virtual permite desenvolver procesos cognitivos para la socialización de la información por interrelación cognitiva, colaboración y asociación, considerándose a estas actividades en un sistema de interconectado (Teixeira \& Martini, 2019). El aprendizaje está concebido como la búsqueda de información, la asociación de conceptos y la predisposición para compartir lo ya adquirido (Siemens, 2004). Estas razones permiten entender en palabras de Downes \& Siemens (Lasso et al., 2017; Teixeira \& Martini, 2019), que los procesos adquisitivos del conocimiento se basan en el intercambio de información, por lo cual, los sujetos utilizan el aprendizaje de otros para incrementar su propio conocimiento y adherirlo como compuestos de información propia.

Las capacidades digitales exigen la orientación y guía de otros sujetos aprendices en las comunidades digitales de aprendizaje. Al respecto, Tourón et al. (2018) consideraron que las competencias digitales son las destrezas o habilidades para emplear la información y llegar al aprendizaje y para la enseñanza. En su sentido pedagógico, las competencias digitales sirven para utilizar los medios informáticos como nexos para generar aprendizajes individuales o colectivos (Garzón et al., 2020; Girón-Escudero et al., 2019; Tourón et al., 2018). Por otro lado, las competencias digitales incluyen procesos específicos para los aprendices en los entornos de aprendizaje: (a) colaboración, (b) asociación, (c) independencia cognitiva (Allueva \& Alejandre, 2019; Brauer, 2019; Brolpito, 2018; Girón-Escudero et al., 2019; Ruíz-Velazco \& Barcena, 2019; Spante et al., 2018). Es decir, los sistemas de aprendizaje y las plataformas digitales en la actualidad buscan que los estudiantes aprendan mediante la colaboración de sus pares, aprender desde la transformación de la información. En un segundo momento, utilizan la información para asociar su sentido objetivo, aplicarlo en situaciones diversas y adherirlas al sistema práctico para llegar al aprendizaje (Brauer, 2019; Brolpito, 2018; Ruíz-Velazco \& Barcena, 2019). En este proceso, los estilos de aprendizaje se adecuan a los ambientes en que el profesorado genera espacios para el conocimiento hasta llegar a la independencia cognitiva.

\section{Competencias digitales en la docencia}

Las competencias digitales, en su sentido cultural, permiten al docente en medios contextuales de emergencia educativa, transformar los entornos de interrelación desde la transformación de los espacios virtuales [códigos, programación, datos], la generación del empoderamiento de grupos aminorados o desatendidos (Brolpito, 2018; Brauer, 2019), cuando 
el profesorado en su grupo más efectivo gestiona procesos efectivos para el aprendizaje en la virtualidad, provocando su calidad y pregnancia constantemente (Tourón et al., 2018). Por lo tanto, las competencias digitales también se transfieren académicamente mediante transposiciones didácticas en la virtualidad (Holguin-Alvarez et al., 2020), De acuerdo a los planteamientos concordantes con Tourón et al. (2018), definen a las competencias digitales como la habilidad para conseguir información, considerándose como medios para la búsqueda del conocimiento (Aristizabal \& Cruz, 2018; Choudrie et al., 2018; Janssen et al., 2013). Sobre su concepción, Janssen et al. (2013) aducen:

La competencia digital implica claramente más que saber cómo utilizar dispositivos y aplicaciones... que está estrechamente relacionado con las habilidades para comunicarse con las TIC, así como con las habilidades de información. El uso sensato y saludable de las TIC requiere conocimientos y actitudes particulares sobre aspectos legales y éticos, privacidad y seguridad, así como comprender el papel de las TIC en la sociedad y una actitud equilibrada hacia la tecnología (p. 480).

En ese sentido, las competencias digitales pueden adquirirse por el conocimiento del uso de los recursos físicos (hardware) o el uso de los recursos digitales (software), por lo que estas competencias incluyen el componente del conocimiento como macro-dimensiones. Esta utilidad, permite comprender como una utilidad más de las competencias en su sentido práctico y técnico para la formación del profesorado (Engen, 2019; Garzón et al., 2020; Levano-Francia et al., 2019; Tourón et al., 2018). Es decir, las competencias o habilidades digitales son capacidades que sirven para adquirir el conocimiento en la práctica, y en el campo educativo, los docentes necesitan de estas competencias para comunicarse, establecer conexiones e impulsar las interacciones cognitivas y/o afectivas (Allueva \& Alejandre, 2019; Brauer, 2019; Brolpito, 2018; Girón-Escudero et al., 2019; Tourón et al., 2018). Por estas razones, se genera asociacionismo del conocimiento en los receptores: el alumnado. La posibilidad de conseguir estas competencias depende de la interacción docente-estudiante, entre los estudiantes, y entre los estudiantes y sus recursos. Esto permite incrementar el perfil académico y el conocimiento de los practicantes (Janssen et al., 2013; Polizzi, 2020). Estas exigencias también han sido declaradas por la Comisión Europea (2009) como parte del perfil digital de los individuos que conforman distintas comunidades digitales, en estas necesitan integrarse a las exigencias de diversos grupos sociales para llegar a desarrollarse.

\section{Aspectos dimensionales de las competencias digitales.}


La dimensión Conocimiento y manejo de la información (Tourón et al., 2018), se refiere a la capacidad para buscar información específica, utilizar los medios de recuperación de información. La fuente de información muchas veces es utilizada de forma poco segura por los alumnos, exponiéndose al peligro. En este sentido, el docente necesita evaluar este componente en la fuente de la información a brindar (Spante et al., 2018). En cuanto a la dimensión Comunicación y colaboración (Comisión Europea, 2009; Tourón et al., 2018), los usuarios pueden generar nuevas fuentes de información para el uso de la habilidad comunicativa. Aquí, la competencia digital debe ser de utilidad recíproca para aprender y comunicar vivencias y aprendizajes (Engen, 2019).

La dimensión Creación de contenidos digitales es la destreza para utilizar los objetivos virtuales de aprendizaje y las herramientas tecnológicas para estructurar y formular componentes de aprendizaje equipados en los entornos virtuales, así se genera autoaprendizaje o aprendizaje colaborativo entre los estudiantes. Estos incrementan sus posibilidades de aprender utilizando los materiales que los propios profesores elaboran (Tourón et al., 2018), como alguna forma de emprendimiento. En el caso de la utilidad de materiales, puede incluirse el uso de telefonía móvil y hardware específico (Choudrie et al., 2018). La dimensión seguridad es la habilidad para aplicar criterios de cuidado y seguridad ante los distintos canales informativos utilizados (Tourón et al., 2018). El concepto se enfoca en la perspectiva educacional, permite que los docentes prevengan los peligros sobre sus propias actividades estructuradas (Aristizabal \& Cruz, 2018; Engen, 2019).

Con esta habilidad, el docente debe salvaguardar la integridad digital de los participantes en los entornos virtuales de aprendizaje (Garzón et al., 2020). Muchos de los peligros a los que se enfrentan están relacionados a tres subdimensiones personales: (a) integridad digital, (b) seguridad de visita, (c) autodominio en redes. Estos peligros pueden evitarse mediante una formación continua a los estudiantes en el entrenamiento constante, ya que son habidos consumidores, a esta actitud pueden apelar los docentes para llegar a la practicidad de estrategias de seguridad digital. En cuanto a la dimensión Resolución de problemas, esto es:

Identificar necesidades y recursos digitales, tomar decisiones a la hora de elegir una herramienta digital apropiada, acorde a la finalidad o necesidad, resolver problemas conceptuales a través de medios digitales, resolver problemas técnicos, uso creativo de la tecnología, actualizar la competencia propia y la de otros (Tourón et al., 2018).

Por lo tanto, el docente impulsa a los sujetos a ampliar sus conocimientos mediante la resolución de problemas en los medios digitales por su cuenta o en colaboración (Aristizabal \& 
Cruz, 2018). Esta habilidad se utiliza tanto para aprender tratando con información extraña (Garzón et al., 2020), muchas veces puede recurrirse a la tecnología artificial (Ocaña-Fernández et al., 2019). Sin embargo, la educación virtual o el proceso para aprender en espacios digitales es totalmente distinto: en tanto el estudiante aprende a escribir por dictado de voz, puede aprender a ubicar el recurso en algún aplicativo o sitio web, utilizarlo sin saber la ruta de acceso y aplicar el registro de voz de forma empírica. Lo cual puede resolverse ante alguna necesidad, sin tener aprendizaje previo significativo.

\section{Hallazgos y problemáticas sobre competencias digitales.}

Entre los antecedentes más cercanos a esta investigación, encontramos que el profesorado presenta problemas para adecuarse al uso de las tecnologías en razón del uso de sus propias competencias digitales (Garzón et al., 2020; Engen, 2019), existe poca proximidad a las actualizaciones de contenidos digitales en cuanto a recursos se refiere. Algunos sujetos presentan problemas para dominar nuevas tecnologías en contextos de gestión privada (Engen, 2019), para lograrlo, el docente debe conocer su entorno, las propiedades o las características que hacen que los sujetos aprendices se embullan en el proceso cognitivo con estas herramientas. En otro contexto escolar, los estudiantes se culturizan al utilizar sus competencias si sus profesores las utilizan en clase (Aristizabal \& Cruz, 2018), mientras los recursos son más atractivos a la vista de ellos, obedecen a sus necesidades y preferencias. Ello ocurre con los usuarios adultos mayores, quienes presentan dificultades para aprender, utilizando únicamente tecnologías computacionales, si logran aprender utilizando sus competencias digitales ante dispositivos móviles inteligentes (Choudrie et al., 2018).

La gamificación se ha considera como el proceso interactivo que desarrolla las competencias digitales con la práctica que realizan los usuarios en sus tareas cotidianas, su practicidad en la docencia permite el desarrollo de habilidades que incrementan el acceso libre ante la cantidad de información existente en la web, de la cual se deben advertir sus beneficios y consecuencias. Al respecto, Sandí y Sanz (2020), han encontrado que diversas condiciones para la formación de competencias digitales: (a) el propósito pedagógico, (b) las rutas o guías para el acceso, (c) el intercambio entre alumnos y docentes sobre el propio juego, (d) los resultados bajo crítica, (e) las estrategias para hacer ludificación del contenido y las actividades. En cambio, Andía et al. (2020), reportan que los profesores presentan competencias digitales ajustadas a sus propios ritmos de enseñanza, en vez de ajustar sus competencias a las capacidades de su alumnado, a pesar de utilizar estrategias como el aula invertida [Flipped Classroom]. 
Aquí cabe resaltar la importancia en el uso de los recursos para desarrollar las habilidades digitales como también, el proceso formativo para estructurar nuevos conocimiento sobre los espacios virtuales. La revisión aquí ha resultado más crucial, debido a la problemática relacionada sobre el uso de la información mediante la aplicación de las estrategias docentes (Andía et al., 2020; Sandí \& Sanz, 2020). En el contexto peruano, más del 98 \% de las escuelas peruanas ostentan los recursos tecnológicos para la atención educativa. Se sabe que más del 25 $\%$ del total de sujetos tiene problemas para consumir datos de Internet con fluidez (INEI, 2020a). Por esta razón nacen algunas preguntas: ¿Será posible lograr empoderamiento de la educación remota en tiempos de pandemia ante la falta de equidad de tipo red-máquina?...Y si se contrarresta esta brecha: ¿será que la adaptación del docente debe hacerse hacia el consumo de los datos o se necesitan de otros factores para generar aprendizajes desde el uso de competencias digitales?

Para responder, en Perdomo et al. (2020), encontramos la falta de investigaciones en competencias digitales, prevaleciendo en la producción científica actual la existencia de trabajos realizados desde la autopercepción de las competencias. Esto es similar al estudio de Cabero-Almenara et al. (2020), en que se obtuvo el análisis del constructo de competencias, el cual es similar al de la Comisión Europea para validar sus aspectos dimensionales (Aristizabal \& Cruz, 2018; Garzón et al., 2020; Tourón et al., 2018). Ante esta parte, asumimos la necesidad del conocimiento en la educación en general. Por otro lado, Buchholz et al. (2020), encontraron que para lograr empoderar la ciudadanía digital, se necesitan manejar medios y acciones constructivas activas, que unifiquen la realidad social con el usuario, como por ejemplo, al utilizar alguna red social. Por lo cual, las competencias digitales deben servir para: (a) involucrarse, (b) participar, (c) reforzar el sentido comunitario digital. En sus evidencias, Fallon (2020) reporta, que las competencias digitales comprendidas como la alfabetización digital, lo cual contrarrestaría la postura procesual. Aquí, tienen lugar los resultados de Tomczyk (2020), quien analizó el componente de seguridad digital como parte de las competencias digitales, por lo que se encontraron firme posición sobre los riesgos en el manejo de instrumentos digitales.

El fin de desarrollar componentes de protección en la educación (resolución de problemas y seguridad digital), es establecer diversos mecanismos de seguridad, para que los estudiantes se sientan seguros en la interacción, la búsqueda y la profundización en los espacios de investigación académica. Se sabe que el acoso a escolares en el Perú se ha incrementado considerablemente desde el año 2015 hasta el 2019, siendo que en la red social Facebook más del $20 \%$ de niños y jóvenes se encuentra acosado de forma verbal, gráfica o video-gráfica, más 
del $50 \%$ de los casos ocurridos ocurren en contextos en que existen mayor consumo de datos en razón de la edad de los habitantes (niños y adolescentes).

Ante el diagnóstico: ¿Por qué es importante conocer y desarrollar las dimensiones relacionadas a la bioseguridad digital desde la práctica docente en los entornos de aprendizaje virtual? La discusión se centra en que el profesorado la desconoce, unos pocos por encontrarse en un periodo de exclusión digital, sin aparcar en redes sociales; y algunos por encontrarse sin tiempo para atender espacios o redes sociales con sus propios estudiantes, realizar búsquedas acompañadas y utilizar blogs en estas búsquedas, ya que asumen que por la edad de sus estudiantes, estos ya ostentan la capacidad de discernimiento de los lugares que visitan. Por lo tanto, el control de visitas es mínimo o casi nulo por parte de los docentes, sobretodo, si estos tienen escaso dominio de las rutas de interacción social mediática de sus alumnos, a pesar de que, existe mayor exigencia cuando se realiza algún modelo de educación remota.

\section{Enfoque humanista de la resiliencia.}

Desde la perspectiva de Carl Rogers, la adquisición de las competencias humanas es vital en cuanto se tenga en cuenta las herramientas humanas y sociales que conlleven a los humanos a superar los obstáculos emergentes. En ese sentido, el humanismo aplicado a la capacidad formativa de la sociedad como un ente formativo per se, implica que la persona utilice recursos emocionales y de autonocimiento para saber sobre sus propias fortalezas y superar dichos problemas. Desde el enfoque humanista, esta integridad, también incluye los objetivos que la persona debe conseguir para satisfacer otras necesidades (Hernández-Peña, 2020; Flores, 2018). Por lo cual, procura satisfacer las necesidades (en ciertos casos, no solo superarlas), implicando la utilización de los recursos sociales para lograr la satisfacción, la productividad en sus labores y la adaptación a distintos medios contextuales-sociales (Gaxiola et al., 2013; Gu \& Day, 2013; Holguin \& Rodríguez, 2020; Mahdiani \& Ungar, 2020; Onofrei, 2015). En muchas estructuras sociales se entiende el sentido de necesidad como la falta de recursos vitales, por lo que en la formación escolar debe generarse el pensamiento proyectivo de vida.

Por otro lado, el enfoque de Maslow (Medeiros et al., 2019; Rodulfo, 2018), propone que las necesidades que el ser humano debe satisfacer, se suscitan en la autoformación. El ser humano busca adquirir potencialidades profesionales, humanas y emocionales para el crecimiento integral (Martinez et al., 2020; Ungar et al., 2020), entre ellos, la resiliencia al optimismo, la autorregulación, la autoevaluación y la autoestima (Gaxiola et al., 2013; Vicente \& Gabari, 2019a). En este sentido, aparece la competencia de la satisfacción de las necesidades 
potenciales para potenciales la cognición y las emociones para la era digital (Mahdiani \& Ungar, 2020). Algunas de estas necesidades se orientan hacia la búsqueda de la sostenibilidad biológica y cognitiva en medios en que necesariamente el ser humano necesita regularse. En la actualidad, los diversos cambios en los medios virtuales, atraen otros problemas que cada vez se vuelven más implícitos e invisibles al ojo humano, como por ejemplo, el secuestro cibernético, Ciberdating, invasión de espacios digitales, falta a la honra personal.

\section{Resiliencia y factores personales.}

La resiliencia se conceptualiza como la competencia humanista, provocada por distintos factores personales. Es la destreza para adoptar distintos recursos humanos para lograr alcanzar metas, superar eventualidades trágicas o para sostener el sentido vital ante el peligro (Gu \& Day, 2013; Mahdiani \& Ungar, 2020; Ungar \& Theron, 2019). Algunas propuestas han considerado como la estrategia para resolver diversos problemas humanos, estas incluyen la resolución de problemas en el peligro, la búsqueda del apoyo emocional para determinar soluciones ante el riesgo (Gaxiola et al., 2013; Ungar \& Theron, 2019; Vicente \& Gabari, $2019^{a}$ ). La resiliencia es la capacidad para la adaptación de los sistemas actitudinales y afectivos de las estructuras sociales (Ungar et al., 2020), es la destreza que configura el enfrentamiento de las debilidades y las amenazas como posibles oportunidades de mejora humana (Gaxiola et al., 2013), aunque se necesitan observar los peligros concretos y reales en las sociedades investigadas.

\section{Aspectos dimensionales de la resiliencia.}

Las características de la personalidad resiliente se basa en el tópico de adaptación humanista hacia la búsqueda de la estabilidad personal. Aquí se considera que el ser humano busca el soporte y equilibrio para la superación de los obstáculos, utiliza herramientas (potencialidades) para solucionar problemas y las percepciones sobre el fracaso (Wagnild \& Young, 1993). Esta posición obedece a una perspectiva adaptacionista de la personalidad hacia las comunidades que presentan dificultades para el progreso social. Los autores Wagnild \& Young (1993), plantearon desde su propuesta las dimensiones: (a) confianza en sí mismo, (b) ecuanimidad, (c) perseverancia, (d) satisfacción personal, (e) sentirse bien solo. Los indicadores que especifican las formas de actuar del ser humano ante los obstáculos se visualizan en la tabla 1. 


\section{Tabla 1}

Aspectos dimensionales de la resiliencia y sus características

\begin{tabular}{ccc}
\hline $\mathrm{N}^{\circ}$. & Dimensiones & Características \\
\hline 1 & Confianza en sí mismo & $\begin{array}{r}\text { Poder autónomo de vida, satisfacción unitaria (personal), } \\
\text { flexibilidad cognitiva y emocional, seguridad vital y } \\
\text { optimismo. }\end{array}$ \\
2 & Ecuanimidad & $\begin{array}{r}\text { Pensamiento visionario (visión futurista de vida), autoestima. } \\
\text { Poder y aceptación para el emprendimiento, automotivación, } \\
\text { voluntad, creatividad voluntaria. }\end{array}$ \\
4 & Perseverancia & Satisfacción personal \\
5 & Sentirse bien solo & $\begin{array}{r}\text { Sentido del humor, sentido del ser o de la existencia, } \\
\text { tolerancia de la existencia y del entorno. } \\
\text { Vivir en soledad y su aceptación vital, pensamiento } \\
\text { discrepante, pensamiento en divergencia. }\end{array}$ \\
\hline
\end{tabular}

Fuente: Caracterización realizada en base a los planteamientos de Wagnild \& Young (1993).

Estas condiciones personales de la resiliencia en el campo educativo, han sido aceptadas como válidas desde la perspectiva de Gu y Day (2013), con la añadidura a la propuesta adaptativa, mencionando que estos factores pueden ser más funcionales de acuerdo a tres aspectos ligados a la identidad docente: (a) convicción (b) vocación. Por otro lado, las propuestas sobre resiliencia en la literatura en español, se dimensionan por Gaxiola et al. (2011), en componentes determinados por factores personales: (a) afrontamiento, (b) actitud positiva, (c) sentido del humor, (d) empatía, (e) flexibilidad, (f) perseverancia, (g) religiosidad, (h) autoeficacia, i) optimismo, (j) orientación a la meta (pp. 76-77). Estas fueron reducidas por análisis empírico factorial a solo 7 factores (Gaxiola et al., 2011), y aceptadas en un estudio posterior con la misma cantidad de factores (Gaxiola et al., 2013). Las dimensiones se acoplan muy bien con la perspectiva educativa de resiliencia psicológica, la cual busca equilibrar el estado de normalidad de los profesores, contrarrestando las influencias de estrés (Gu \& Day, 2013; Li et al., 2019), esto les permite ser menos vulnerables hacia dichas influencias y generar autoeficacia.

En la resiliencia docente aceptamos las propuestas de Li et al. (2019), quienes plantean como sus componentes dimensionales: (a) Compromiso y motivación docente, (b) sentido de autoeficacia, (c) realización laboral. Respecto a la primera, es el concepto sobre la competencia que involucra la motivación, el rendimiento, la persistencia y el compromiso por el cambio constante en la función docente (p. 146). En el sentido de la autoeficacia, los indicadores caracterizan a este componente como la percepción para dar cuenta de las propias oportunidades 
y defectos para encontrar bienestar en el proceso de gestión docente, aceptarlos, incrementar algunos y aminorar otros que guarden cierta relación, ya sea positiva o negativa, con su propio ejercicio profesional (p. 147). La realización laboral configura el componente del profesorado en características como optimismo, pasión, sentido actitudinal, aspiración personal y el sentido de capacidad ante su propio ejercicio, de acuerdo a los objetivos de la profesión, e incluye la satisfacción por el cumplimiento de las responsabilidades profesionales y personales.

\section{Hallazgos y problemáticas relacionadas con la resiliencia.}

En la literatura internacional, Clará (2017) encontró que las adversidades evaluadas y reevaluadas por el profesorado permiten superar sus estados de adversidad desde el sufrimiento del dolor (no riesgo). Esto también se ha dado en el estudio de Chesak et al. (2019), quien desde el manejo del sufrimiento basado en Mindfulness encontró que los docentes aminoran la ansiedad, incrementando la gratitud, la satisfacción y la felicidad propia (subjetiva). Por otro lado, en Ainsworth and Oldfield (2019), los resultados que avalan predictores de la resiliencia en docentes (la satisfacción en el trabajo, el estrés laboral, el bienestar), por acción de factores sociales (contextuales), los que influyen más que los factores personales, por leves diferencias. Entre los resultados más actuales, encontramos a Galindo-Domínguez et al. (2020), los cuales determinaron a la resiliencia como una variable independiente, la cual aporta (pero no influye directamente) en la autoeficacia y aminora el burnout.

En la literatura en español, hallamos en Vicente y Gabari (2019a) que la regulación emocional (como parte de la resiliencia) permite que los profesores se comprometan con sus puestos de trabajo, el ajuste de su estilo de vida, sus afectos y autorregulación emocional para ser eficientes de forma sostenible, a diferencia de los menos resilientes, quienes son más vulnerables en sus labores. Esto también coincide con lo encontrado por Vicente y Gabari (2019b): existen menos probabilidades de ser docentes más vulnerables ante el burnout siempre cuando hay altos niveles de resiliencia. Sin embargo, Vicente y Gabari (2019c) hallaron la relación entre la actitud positiva basada en el optimismo y la resiliencia en sus dimensiones confianza y persistencia. En otros estudios similares, encontraron que altos puntajes en el agotamiento emocional y a la falta de personalidad (personalización), disminuyen el potencial de la resiliencia en la labor profesional (Tacca \& Tacca, 2019). Los resultados en lengua extranjera (Ainsworth \& Oldfield, 2019; Clará, 2017; Chesak et al., 2019; Galindo-Domínguez et al., 2020), rescatan aspectos sociales del profesorado como determinantes de las herramientas para utilizar la resiliencia ante su tarea educativa, aunque no se denotan el análisis de los datos personales en contextos determinantes (Gaxiola et al., 2013; Ungar \& Theron, 2019). 
En relación a estos hallazgos, se puede asumir la necesidad de plantear la resiliencia como un aspecto dimensional formativo del perfil docente en instituciones educativas de Latinoamérica y en común, en las peruanas. La necesidad radica en el peligro que vive la comunidad peruana, y entre los cuales, los profesores se encuentran afectados por los factores sociales, y más aún, los factores laborales, tanto como los personales. Al respecto, se conoce que hasta antes de la etapa pandémica, existieron más del año 2019, 57.552 casos sobre violencia psicológica, y más de 51.000 casos de violencia física (Ministerio de la Mujer y Poblaciones Vulnerables, MIMP, 2019), y el $96 \%$ de las mujeres y el $4 \%$ de los varones ha sufrido algún tipo de violencia, específicamente debido a la convivencia en contextos vulnerables.

Además, la percepción de violencia ha aumentado considerablemente hasta la mitad del año 2020, a pesar de encontrarnos en períodos de aislamiento social, ya que casi el $85 \%$ del total de ciudadanos cree que estará en peligro en su sociedad o entorno próximo (Instituto Nacional de Estadística del Perú, INEI, 2020b, 2020c). Estas cifras anuncian que el profesorado necesita de ciertas competencias humanas tales como la resiliencia, convirtiéndola en el sostenimiento espiritual, emocional y social en la relación del individuo con su entorno. En algunos casos, los docentes necesitan de herramientas de afrontamiento de la realidad, incrementan su resiliencia en grupos únicos quienes también utilizan sus competencias digitales de forma efectiva, establecen interacciones saludables (Acevedo-Duque, et al., 2020; MartínezAngoa, 2020). En otros casos, se fortalecen los vínculos sociales entre el profesorado y el alumnado acorde a la resiliencia distribuida ante presiones académicas (Martínez-Angoa, 2020).

\section{Discusión}

Respecto a las competencias digitales, hemos encontrado similitudes esenciales en su concepto y fundamentación, pues la mayoría de autores parte desde el enfoque DigComp planteado en diversas investigaciones en relación a los aportes de la Unión Europea e investigadores de dicho contexto. Su definición se ha centrado en el dominio de la habilidad tanto como el conocimiento sobre la información encontrada en la Internet, para utilizarla hacia una adaptación a la comunidad digital (Aristizabal \& Cruz, 2018; Andía et al., 2020; Choudrie et al., 2018; Garzón et al., 2020; Engen, 2019). Sin embargo, en el campo educativo ya se consideran estas capacidades aplicadas a la enseñanza, con el fin de generar espacios de productividad escolar, interactividad y aprendizaje colaborativo entre el docente y el estudiante (Holguin-Alvarez et al., 2021a; Instefjord et al., 2017; Lucas et al., 2021; Sandí \& Sanz, 2020; 
Wild \& Schulze, 2021; Zhao et al). Respecto a esto se debe separar el concepto para integrarlo en la escuela como una forma de autoformación del profesional docente, más bien como una exigencia secuencial en el proceso pedagógico, más que el único hecho de considerarlo como parte del perfil docente (Hämäläinen et al., 2021; Lucas et al., 2021). El contexto pandémico ha enseñado que es necesaria la capacitación en distintas áreas, tanto en la emocional como en la formación digital, el desarrollo de la práctica docente depende mucho del desarrollo que se busque en los estudiantes.

En cuanto a la utilidad de estas competencias en el área educacional, se ha determinado que las capacidades para dominar la información y los recursos tecnológicos [informarse, buscar recursos, brindar seguridad, resolver problemas], se adecuan a los conceptos formativos desde el enfoque de aprendizaje por competencias, cuyo origen se ha visto marcado por la aparición del enfoque conectivista, constructivista y socioemocional de las teorías educativas, por lo cual, es esencial para el docente producir estrategias que permitan utilizar la información como medio de investigación en los estudiantes, y la generación de aprendizajes colaborativos y autónomos, así como se ha hallado en algunas investigaciones (Aristizabal \& Cruz, 2018; Holguin-Alvarez et al., 2021a; Sandí \& Sanz, 2020). Al respecto, es importante que los conceptos de competencia digital puedan permitir que los docentes conozcan qué aprender, ello lo pueden agregar a los contenidos curriculares del plan de trabajo con el cual se dirigirá la institución educativa. La importancia de este punto recae en que muchas veces los profesores y estudiantes practican y desarrollan las habilidades sin conocer cuáles, es insuficiente la practicidad cuando se desconoce el camino por el que se aprende, así como lo aducen Lucas et al. (2021): para empoderar a los alumnos en competencias digitales, el compromiso docente para aprenderlas es un medio, pero comprenderlas también sería un co-aprendizaje.

Estas perspectivas son exigencias actuales respecto a las competencias digitales, conocerlas, definirlas y dimensionarlas permitirá responder a las exigencias y coyunturas contemporáneas, tal como lo afirma Lucas et al. (2021), en concordancia con Buchholz et al. (2020) y Fallon (2020), puesto que la digitalización del mundo está incrementando, pero a la vez, transformando las formas de comunicación, los sentimientos, los estilos para aprender y hasta las formas de trabajar; se hace más recurrente y a su vez, más emergente la necesidad de formar no solo a los individuos que desarrollen el país, siendo estos los más talentosos, sino también a aquellos que con poco talento para integrarse a la comunidad digital, puedan desenvolverse tan bien como aquellos para poder sobrevivir satisfaciendo sus necesidades básicas. 
En relación a la resiliencia, el concepto basado en la capacidad de afrontamiento psicológico de la persona sobre las vicisitudes emergentes en la vida (Ainsworth \& Oldfield, 2019; Gaxiola et al., 2013; Ungar \& Theron, 2019), ha virado hacia la comprensión de una resiliencia más organizacional en las instituciones educativas, por cuanto se le conoce como la capacidad para prevenir los desastres de las instituciones ante el pre-abordaje y procesamiento de los obstáculos emergentes en el proceso laboral (Duchek, 2019), esto compromete a los empleados como integrantes de estas organizaciones o como la parte integrada de las empresas inteligentes (Andersson et al., 2019; Duchek, 2017), siendo también resilientes. En este sentido, el fundamento de la resiliencia para el profesorado se ha centrado en tres perspectivas: (a) psicobiológica de Ungar, (b) humanista de Rogers, (c) satisfactoria de Maslow. Desde la primera, podríamos entender que los profesores utilicen sus pensamientos y los elementos de su organismo para satisfacer necesidades que permitan desviar los obstáculos, esto se comprende como el solapamiento de los estados de crisis para echarlos al olvido. Por lo general, en personas con necesidades contextuales más profundas, se suele recordar la crisis, lo cual cimenta paulatinamente el fracaso laboral.

Este concepto se diferencia aquel que los docentes utilizarían para humanizar el sentido de la pérdida o un evento fortuito, concentrándose en los factores contextuales externos más que personales. Es así que el profesorado necesita tener en cuenta los recursos que habilitarían más habilidades en ellos para lograr desarrollar con éxito la pedagogía y su gestión curricular, más aún en contextos contrarios como el pandémico, por cuanto necesita humanizar los recursos no propios de su integridad biológica. Esto se ha encontrado en otros contextos en que la satisfacción, el optimismo y la seguridad se acrecentó en personas que pasaron por eventos desastrosos, pero se valieron de otros recursos externos (brindados por otros) como la gratitud o el agradecimiento (Ainsworth \& Oldfield, 2019; Chesak et al., 2019). En muchos casos el agotamiento y el estrés suele generar pérdida de peso de la variable resiliencia (Ainsworth \& Oldfield, 2019), ante los obstáculos en personas que trabajan en organizaciones que descuidan el bienestar de sus trabajadores.

Entre las limitaciones más importantes, se sitúan las relacionadas a la selectividad por tiempo, ya que significativo número de referencias sobre resiliencia se encontró con antigüedad mayor a 8 o 10 años, lo cual empuja a revisar este constructo y su relación histórica con las ciencias de la psicología o la psicología educacional en estudios de revisión sistemática a posteriori. Por otro lado, la centralización de temáticas respecto a las competencias digitales en los artículos científicos, limitó al estudio a reportar en su mayoría, investigaciones basadas en la autopercepción, aunque existe un número básico, pero inicial de las competencias desde los 
enfoques tecnológicos, consumistas, y otros basados netamente en alfabetización. Por último, el acceso a la literatura grande en textos de libro ubicados en la base de Scopus, es una limitación institucional la cual debería gestionarse para ampliar el espectro hacia estudios fundamentales o que permitan desarrollar teorías sustentadas de enfoque cualitativo.

Finalmente, es crucial que el currículo formativo de la universidad en el proceso del docente en formación sea más satisfactorio, desde el aprendizaje de teorías educativas y desde la práctica pre profesional, con el fin de fortalecer la identidad docente sostenible. Basándonos en un esquema de satisfacción de necesidades humanas, algunas variables como la regulación emocional, el estilo de vida, la potenciación de la personalidad; y otras características personales en el profesorado (Vicente \& Gabari, 20219a; 2019b; 2019c; Tacca \& Tacca, 2019), permiten incrementar las defensas humanísticas de la persona ante el sufrimiento, ya que el sufrimiento se considera un elemento vital para el autodesarrollo (Ainsworth \& Oldfield, 2019; Ungar \& Theron, 2019), aprender desde su dominio permite que las competencias para la sostenibilidad institucional sean más provechosas, así como el cambio emocional de la persona para hacer más sostenibles sus procesos vitales. Ante este hecho, podemos aseverar que el concepto de resiliencia se orienta hacia el uso de factores protectores personales, más internos y psicológicos para lograr una defensa anticipada y personalista de tipo emocional, para abordar eventos desastrosos; en contraposición a las ideas de una resiliencia psico-biológica o humanista.

\section{Conclusiones}

En cuanto a las competencias digitales, se realizó el acercamiento hacia el conectivismo y el constructivismo digital. El conectivismo aplicado al ámbito educacional, permite conocer cuáles son las estrategias de los profesores para utilizar la información como medio de enseñanza, y establecer los conocimientos que tienen sobre ella para la tarea docente efectiva. Respecto al enfoque de la resiliencia, determinamos su concepto allegado a la perspectiva humanista de la personalidad, como también, hacia el enfoque basado en la teoría de las necesidades humanas. En principio, es humanista en su sentido práctico, vivencial y actitudinal, puesto que genera en el docente la capacidad de respuesta, soporte, evaluación y retroalimentación para lograr superar los momentos de pérdida, desesperación y falta. Estos conceptos, usualmente negativos en la tarea docente, son aquellos que permiten despertar esta competencia.

Finalmente, cabe destacar la riqueza en investigación de estos constructos dedicados hacia la psicología educacional o psicología positiva aplicada a la educación, ya que resultan 
valiosos los aportes para generar nuevas definiciones e ideas caracterizables en la nueva sociedad del conocimiento. Por otro lado, es sugerente romper con el paradigma psicológico aplicado únicamente a las investigaciones en personalidad, para ubicar el centro de producción científica de la resiliencia en el campo educativo.

\section{Referencias}

Acevedo-Duque, Á.; Arguello, A. J.; Pineda, B.G.; \& Turcios, P.W. (2020). Competencias del docente en educación online en tiempo de COVID-19: Universidades Publicas de Honduras. Revista de Ciencias Sociales, 26, 206-224. http://dx.doi.org/10.31876/rcs.v26i0.34123

Ainsworth, S. \& Oldfield, J. (2019). Quantifying teacher resilience: Context matters. Teaching and Teacher Education, 82, 117-128. https://doi.org/10.1016/j.tate.2019.03.012

Allueva, P. \& Alejandre, M. (2019). Enfoques y experiencias de innovación educativa con TIC en educación superior. Prensas de la Universidad de Zaragoza.

Andía, L. A.; Campión, R. S.; \& Sota, J. M. (2020). ¿Estamos técnicamente preparados para el flipped classroom? Un análisis de las competencias digitales de los profesores en España. Contextos educativos. Revista de Educación, 25, 275-311. https://doi.org/10.18172/con.4218

Andersson, T.; Cäker, M.; Tengblad; S. \& Wickelgren, M. (2019). Building traits for organizational resilience through balancing organizational structures. Scandinavian Journal of Management, 35 (1), 36-45. https://doi.org/10.1016/j.scaman.2019.01.001

Aristizabal, P. \& Cruz, E. (2018). Development of digital competence in the initial teacher education of early childhood education. Pixel-bit-Revista de medios y educacion, 52, 97-110. https://doi.org/10.12795/pixelbit.2018.i52.07

Brauer, S. (2019). Digital Open Badge-Driven Learning-Competence-based Professional Development for Vocational Teachers. Acta Universitatis Lapponiensis 380, University of Lapland Printing Centre, Rovaniemi, 380. https://lauda.ulapland.fi/bitstream/handle/10024/63609/Brauer_Sanna_ActaE_247pdf A.pdf?sequence $=1 \&$ isAllowed $=\mathrm{y}$

Brolpito, A. (2018). Digital Skills and Competence, and Digital and Online Learning. European Training Foundation. https://eufordigital.eu/library/digital-skills-and-competence-anddigital-and-online-learning/ 
Buchholz, B. A.; DeHart, J.; \& Moorman, G. (2020). Digital Citizenship During a Global Pandemic: Moving Beyond Digital Literacy. Journal of Adolescent \& Adult Literacy, 64 (1), 11-17. https://doi.org/10.1002/jaal.1076

Cabero-Almenara, J.; Barroso-Osuna, J.; Palacios-Rodríguez, A.; \& Llorente-Cejudo, C. (2020). Marcos de Competencias Digitales para docentes universitarios: su evaluación a través del coeficiente competencia experta. Revista Electrónica Interuniversitaria de Formación del Profesorado, 23 (2), https://doi.org/10.6018/reifop.413601

Comisión Europea (2009). Marco estratégico Educación y Formación 2020 (ET2020). España.

Chesak, S. S.; Khalsa, T. K.; Bhagra, A.; Jenkins, S. M.; Bauer, B. A.; \& Sood, A. (2019). Stress Management and Resiliency Training for public school teachers and staff: A novel intervention to enhance resilience and positively impact student interactions. Complementary Therapies in Clinical Practice, 37, 32-38. https://doi.org/10.1016/j.ctcp.2019.08.001

Choudrie, J.; Pheeraphuttharangkoon, S.; \& Davari, S. (2018). The digital divide and older adult population adoption, use and diffusion of mobile phones: A Quantitative Study. Information Systems Frontiers, 21 (98), 1-29. https://doi.org/10.1007/s10796-018$\underline{9875-2}$

Clará, M. (2017). Teacher resilience and meaning transformation: How teachers reappraise situations of adversity. Teaching and Teacher Education, 63, 82-91. https://doi.org/10.1016/j.tate.2016.12.010

De Jager, T.; Coetzee, M. J.; Maulana, R.; Helms-Lorenz, M.; \& Van de Grift, W. (2017). Profile of South African secondary-school teachers' teaching quality: evaluation of teaching practices using an observation instrument. Educational Studies, 43 (4), 410429. https://doi.org/10.1080/03055698.2017.1292457

Díaz, V.P. (2009). Metodología de la investigación científica y bioestadística para profesionales y estudiantes de Ciencias de la Salud. RIL editores.

Duchek, S. (2019). Organizational resilience: a capability-based conceptualization. Business Research, 13, 215-246. https://doi.org/10.1007/s40685-019-0085-7

Duchek, S. (2017). Entrepreneurial resilience: a biographical analysis of successful entrepreneurs. International Entrepreneurship and Management Journal, 14, 429-455. https://doi.org/10.1007/s11365-017-0467-2

Engen, B. (2019). Understanding social and cultural aspects of teachers' digital competencies. Comunicar, 27 (61), 9-19. https://doi.org/10.3916/C61-2019-01 
Fallon, G. (2020). From digital literacy to digital competence: the teacher digital competency (TDC) framework. Educational Technology Research and Development, 68, 24492472. https://doi.org/10.1007/s11423-020-09767-4

Flores, J.A. (2018). Resiliencia, depresión y agresividad en estudiantes del 5 to año de educación secundaria, 2017-Rímac. [Tesis doctoral]. Universidad César Vallejo, Lima, Perú.

Galindo-Domínguez, H.; Pegalajar, M.; \& Uriarte, J.D.D. (2020). Efecto mediador y moderador de la resiliencia entre la autoeficacia y el burnout entre el profesorado universitario de ciencias sociales y legales. Revista de Psicodidáctica, 25 (2), 127-135. https://doi.org/10.1016/j.psicod.2020.04.002

Garzón, A.; Sola, M.; Ortega, M.; Marín, M. \& Gómez, G. (2020). Teacher Training in Lifelong Learning-The Importance of Digital Competence in the Encouragement of Teaching Innovation. Sustainability Journal, 12 (7), 1 - 13. https://doi.org/10.3390/su12072852

Gaxiola, J. C.; González, S.; Domínguez, M.; \& Gaxiola, E. (2013). Autorregulación, metas y rendimiento académico en bachilleres con disposiciones resilientes y no resilientes. Revista Interamericana de Psicología/Interamerican Journal of Psychology, 47 (1), 71 82. https://doi.org/10.30849/rip/ijp.v47i1.203

Gaxiola, J.C.; Frías, M.; Hurtado, M.F.; Salcido, L.C.; Figueroa, M. (2011). Validación del inventario de resiliencia (IRES) en una muestra del noreste de México. Enseñanza e $\begin{array}{lllll}\text { Investigación en } & \text { psicología, } & 16 & \text { (1), }\end{array}$ http://www.redalyc.org/articulo.oa?id=29215963006

Girón-Escudero, V.; Cózar-Gutiérrez, R.; \& González-Calero, J. A. (2019). Análisis de la autopercepción sobre el nivel de competencia digital docente en la formación inicial de maestros/as. Revista Electrónica Interuniversitaria de Formación del Profesorado, 22 (3), 193-218. https://doi.org/10.6018/reifop.373421

Gu, Q. \& Day, C. (2013). Challenges to teacher resilience: conditions count. British $\begin{array}{lllll}\text { Educational } & \text { Research } & \text { Journal, } & 39 & \text { (1), }\end{array}$ http://dx.doi.org/10.1080/01411926.2011.623152

Hämäläinen, R.; Nissinen, K.; Mannonen, J.; Lämsä, J.; Leino, K.; \& Taajamo, M. (2021). Understanding teaching professionals' digital competence: What do PIAAC and TALIS reveal about technology-related skills, attitudes, and knowledge? Computers in Human Behavior, 117, 106672. https://doi.org/10.1016/j.chb.2020.106672

Hernández, A.A.; Ramos, M.P.; Placencia, B.M.; Indacochea, B.; Quimis, A.J.; \& Moreno, L.A. (2018). Metodología de la investigación científica. 3Ciencias. 
Hernández-Peña, H.; Aguirre-Martinez, G.; Estay-Sepúlveda, J.G.; Lagomarsino-Montoya, M.; Mansilla-Sepúlveda, J.; \& Ganga-Contreras, F. (2020). La era digital comprendida desde la Psicología humanista. Revista Costarricense de Psicología, 39 (1), 35-53. http://www.rcps-cr.org/openjournal/index.php/RCPs/article/view/144

Holguin-Alvarez, J.; Gara-Rodríguez, P.; Amasifuén-Sánchez, V.; \& Huaita, D. M.; Luza, F. F.; Cruz-Montero, J.; \& Ledesma-Pérez, F. (2021a). Digital Competences in the Elderly and University Students: Didactic Interaction from the Use of Social Networks. International Journal of Emerging Technologies in Learning, 16 (04), 188-200. https://doi.org/10.3991/ijet.v16i04.18519

Holguin-Alvarez, J.; Apaza-Quispe, J.; Ruiz, J.M.; \& Picoy, J.A. (2021b). Competencias digitales en directivos y profesores en el contexto de educación remota del año 2020. Revista Venezolana de Gerencia, $26 \quad$ (94), 623-643. https://doi.org/10.52080/rvgluzv26n94.10

Holguin-Alvarez, J.; Manrique-Alvarez, G.; Apaza-Quispe, J.; \& Romero-Hermoza, R. (2020). Digital Competences in the Social Media Program for Older Adults in Vulnerable Contexts. International Journal of Scientific and Technology Research, 9 (5), 228-232. https://www.ijstr.org/final-print/may2020/Digital-Competences-In-The-Social-MediaProgram-For-Older-Adults-In-Vulnerable-Contexts.pdf

Holguin, J. \& Rodríguez, M. (2020). Proactividad y resiliencia en estudiantes emprendedores de Lima. Propósitos y $\quad$ Representaciones, $8 \quad$ (2), e367. http://dx.doi.org/10.20511/pyr2020.v8n2.367

Instefjord, E.J. \& Munthe, E. (2017). Educating digitally competent teachers: A study of integration of professional digital competence in teacher education. Teaching and Teacher Education, 67, 37-45. https://doi.org/10.1016/j.tate.2017.05.016

Instituto Nacional de Estadística e Informática del Perú (INEI, 2020a). Más de medio millón de maestros en el Perú celebran su día. http://m.inei.gob.pe/prensa/noticias/mas-demedio-millon-de-maestros-en-el-peru-celebran-su-dia-9833/

Instituto Nacional de Estadística e Informática del Perú (INEI, 2020b). Principales indicadores de seguridad ciudadana a nivel regional. Semestre Móvil, Noviembre 2019-Abril, 2020. INEI.

Instituto Nacional de Estadística e Informática del Perú (INEI, 2020c). Estadísticas de seguridad ciudadana-Informe técnico. Marzo-Agosto, 2020-Boletín $\mathrm{N}^{\circ}$ 05-Octubre, 2020. INEI. 
Janssen, J.; Stoyanov, S.; Ferrari, A.; Punie, Y.; Pannekeet, K.; \& Sloep, P. (2013). Experts' views on digital competence: Commonalities and differences. Computers \& Education, 68, 473-481. https://doi.org/10.1016/j.compedu.2013.06.008

Lasso, E. P.; Munévar, P. A.; Rivera, J. A.; \& Sabogal, A. (2017). Estado del arte sobre la articulación de modelos enfoques y sistemas en educación virtual. Libros Universidad $\begin{array}{lllll}\text { Nacional Abierta } & \text { y }\end{array}$ https://hemeroteca.unad.edu.co/index.php/book/article/view/1969

Levano-Francia, L.; Sanchez, S.; Guillén-Aparicio, P.; Tello-Cabello, S.; Herrera-Paico, N.; \& Collantes-Inga, Z. (2019). Competencias digitales y educación. Propósitos y representaciones, 7 (2), 569-588. http://dx.doi.org/10.20511/pyr2019.v7n2.329

Li, Q.; Gu, Q.; \& He, W. (2019). Resilience of Chinese Teachers: Why Perceived Work Conditions and Relational Trust Matter. Measurement: Interdisciplinary Research and Perspectives, 17 (3), 143-159. https://doi.org/10.1080/15366367.2019.1588593

Lucas, M.; Bem-Haja, P.; Siddiq, F.; Moreira, A.; \& Redecker, C. (2021). The relation between in-service teachers' digital competence and personal and contextual factors: What matters most? Computers \& Education, 160, 104052. https://doi.org/10.1016/j.compedu.2020.104052

Mahdiani, H. \& Ungar, M. (2020, March 12). Can Biomedical and Cognitive Enhancement Increase Psychological Resilience?. Canadian Psychology. Advance online publication. http://dx.doi.org/10.1037/cap0000217

Martínez-Angoa, T. (2020). Resiliencia docente: un estudio de caso en una preparatoria universitaria. Revista de educación de la Rediech, 11, 1-20. https://doi.org/10.33010/ie_rie_rediech.v11i0.882

Martínez, N.; Villalobos, J.; \& Machado, M. (2020). Autorrealización: elaboración de la escala ADO-20 para su evaluación en docentes. Revista Estudios en Educación, 3 (4), 181 211. http://ojs.umc.cl/index.php/estudioseneducacion/article/view/92/69

Medeiros, C., Diógenes, M., Magalhães, M., Veloso, G., Evangelista, M. y Cunha, M. (2019). Jerarquía de Necesidades de Maslow: Validación de un Instrumento. Psicologia: Ciência e Profissão, 39, 1-13. http://dx.doi.org/10.1590/1982-3703003183408

Ministerio de Educación del Perú-Estadística de la Calidad Educativa [ESCALE] (2019). Encuesta Nacional a Instituciones Educativas - ENEDU. http://escale.minedu.gob.pe/192

Ministerio de la Mujer y Poblaciones Vulnerables (2019). Informe estadístico. Violencia en cifras. Boletín N. 12-Diciembre, 2019. 
Ministerio de Salud del Perú (Minsa, 22 de junio de 2021). Sala Situacional del Covid-19 Perú. (Visualizado: 25 de junio de 2021). https://covid19.minsa.gob.pe/sala_situacional.asp

Mithani, M.A.; Gopalakrishnan, S.; \& Santoro, M.D. (2021). Does exposure to a traumatic event make organizations resilient? Long Range Planning, 54 (3), 102031. https://doi.org/10.1016/j.lrp.2020.102031

Ocaña-Fernández, Y.; Valenzuela-Fernández, L.A.; \& Garro-Aburto, L.L. (2019). Inteligencia artificial y sus implicaciones en la educación superior. Propósitos y representaciones, 7 (2), 536-568. http://dx.doi.org/10.20511/pyr2019.v7n2.274

Onofrei, S.G. (2015). Teaching Using New Technologies and Students Resilience. Acta Didactica Napocensia, 8(2), 33-42. http://oaji.net/journal-detail.html?number=551

Organización de las Naciones Unidas para la Educación, la Ciencia y la Cultura-Unesco (4 de abril de 2021). Reforzar el aprendizaje y las capacidades digitales en los países más poblados del mundo para estimular la recuperación de la educación. https://es.unesco.org/news/reforzar-aprendizaje-y-capacidades-digitales-paises-maspoblados-del-mundo-estimular

Perdomo, B.; González, O.; \& Barrutia, I. (2020). Competencias digitales en docentes universitarios: una revisión sistemática de la literatura. EDMETIC, 9 (2), 92-115. https://doi.org/10.21071/edmetic.v9i2.12796

Polizzi, G. (2020). Digital literacy and the national curriculum for England: Learning from how the experts engage with and evaluate online content. Computers \& Education, 152,103859. https://doi.org/10.1016/j.compedu.2020.103859

Rodulfo, J. (2018). ¿Por Qué Maslow?: Como usar su Teoría para permanecer en el Poder para Siempre. Rodulfo.

Rudas, J.S.; Gómez, L.M.; \& Toro, A.O. (2013). Revisión sistemática de la literatura. Caso de estudio: Modelamiento de un par deslizante con fines de predecir desgaste. Prospectiva, 11 (1), 50-58. http://www.redalyc.org/articulo.oa?id=496250735006

Ruíz-Velazco, S. \& Barcena, L. (2019). Edutecnología y Aprendizaje 4.0. SOMECE.

Rupprech, S.; Koole, W.; Chaskalson, M.; Tamdjidi,C.; \& West, M. (2019). Running too far ahead? Towards a broader understanding of mindfulness in organisations. Current Opinion in Psychology, 28, 32-36. https://doi.org/10.1016/j.copsyc.2018.10.007

Sánchez-Cruzado, C.; Santiago, R.; \& Sánchez-Compaña, M.T. (2021). Teacher Digital Literacy: The Indisputable Challenge after COVID-19. Sustainability, 13 (4), 1858. https://doi.org/10.3390/su13041858 
Sandí, J. C. \& Sanz, C. V. (2020). Juegos serios para potenciar la adquisición de competencias digitales en la formación del profesorado. Revista Educación, 44(1), 2215-2644. http://dx.doi.org/10.15517/revedu.v44i1.37228

Siemens, G. (2014). Connectivism: A Learning Theory for the Digital Age. [elearnspace.org]. http://citeseerx.ist.psu.edu/viewdoc/download?doi=10.1.1.1089.2000\&rep=rep1\&type $=$ pdf

Spante, M.; Hashemi, S.; Lundin, M.; \& Algers, A. (2018). Digital Competence and Digital Literacy in Higher Education Research: Systematic Review of Concept Use. Cogent Education Journal, 5 (1). 1-21. https://doi.org/10.1080/2331186X.2018.1519143

Tacca, D. R. \& Tacca, A. L. (2019). Síndrome de Burnout y resiliencia en profesores peruanos. Revista de Psicología, 22, 11-30. http://www.scielo.org.bo/scielo.php?pid=S2223$\underline{30322019000200003 \& \text { script }=\text { sci_abstract\&tlng=pt }}$

Teixeira, D. \& Martini, S.C. (2019). Conectivismo Pedagógico: novas formas de ensinar e aprender no século XXI. Revista Thema, 16 (4), 1012-1025. https://doi.org/10.15536/thema.V16.2019.1012-1025.1583

Tomczyk, L. (2019). Skills in the area of digital safety as a key component of digital literacy among teachers. Education and Information Technologies, 25, 471-486. https://doi.org/10.1007/s10639-019-09980-6

Tondeur, J.; Aesaert, K.; Pynoo, B.; Van Braak, J.; Fraeyman, N.; \& Erstad, O. (2015). Developing a validated instrument to measure preservice teachers' ICT competencies: Meeting the demands of the 21st century. British Journal of Educational Technology, 48 (2), 462-472. https://doi.org/10.1111/bjet.12380

Tourón, J.; Martín, D.; Navarro, E.; Pradas, S.; \& Íñigo, V. (2018). Validación de constructo de un instrumento para medir la competencia digital docente de los profesores (CDD). Revista Española de Pedagogía, 76 (269), 25-54. https://doi.org/10.22550/REP76-12018-02

Ungar, M. \& Theron, L. (2019). Resilience and mental health: how multisystemic processes contribute to positive outcomes. Lancet Psychiatry, 7 (5), 441-448. https://doi.org/10.1016/S2215-0366(19)30434-1

Ungar, M., J.; McRuer, X.; Liu, L.; Theron, D.; Blais, D.; \& Schnurr, M. (2020). Socialecological resilience through a biocultural lens: a participatory methodology to support global targets and local priorities. Ecology and Society, 25 (3):8. https://doi.org/10.5751/ES-11621-250308 
Vicente, M. I. \& Gabari, M. I. (2019a). La resiliencia como factor protector del estrés crónico en docentes. European Journal of Investigation in Health, Psychology and Education, 9 (3), 159-175. https://doi.org/10.30552/ejihpe.v9i3.332

Vicente, M.I. \& Gabari, M.I. (2019b). Burnout y Factores de Resiliencia en Docentes de Educación Secundaria. International Journal of Sociology of Education, 8 (2), 127-152. http://dx.doi.org/10.17583/rise.2019.3987

Vicente, M.I. \& Gabari, M.I. (2019c). Emociones positivas: una herramienta psicológica que contribuye al proceso de resiliencia en los profesionales de la educación. International Journal of Developmental and Educational Psychology, 4 (1), 159-172. https://doi.org/10.17060/ijodaep.2019.n1.v4.1539

Villalobos-Otayza, A.; Vela-Alfaro, F.M.; Wiegering-Gianoli, D.; \& Robles-Alfaro, R.A. (2021). Level of resilience and depressive symptoms in medical interns in Peru. Educación Médica, 22 (1), 14-19. https://doi.org/10.1016/j.edumed.2018.12.006

Wagnild, G.M. \& Young, H.M. (1993). Development and Psychometric Evaluation of the Resilience Scale. Journal of Nursing Measurement, 1 (2), 165-178.

Wild, S. \& Schulze, L. (2021). Re-evaluation of the D21-digital-index assessment instrument for measuring higher-level digital competences. Studies in Educational Evaluation, 68, 100981. https://doi.org/10.1016/j.stueduc.2021.100981

Wingerden, J.V. \& Poell, R.F. (2019). Meaningful work and resilience among teachers: The mediating role of work engagement and job crafting. PloS ONE, 14 (9), e0222518. https://doi.org/10.1371/journal.pone.0222518

Zhao, Y.; Pinto, A.M.; \& Sánchez, M.C. (2021). Digital competence in higher education research: A systematic literature review. Computers \& Education, 168, 104212. https://doi.org/10.1016/j.compedu.2021.104212 